\title{
Decarboxylative Coupling by Photoredox and Chiral Nickel Catalysis
}

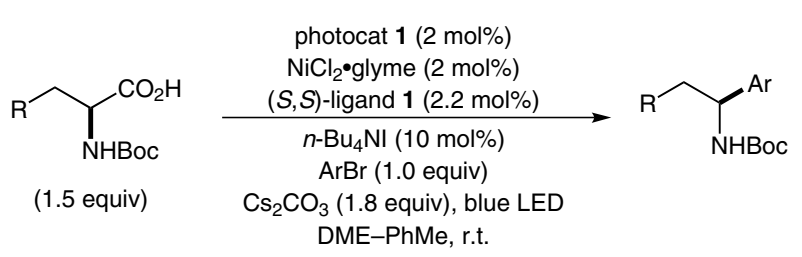

Design plan:<smiles>[R]C(N)C(=O)O</smiles>
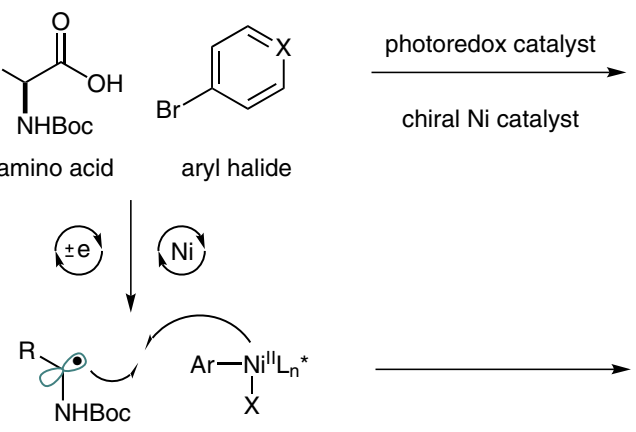

enantioenriched benzylic amine

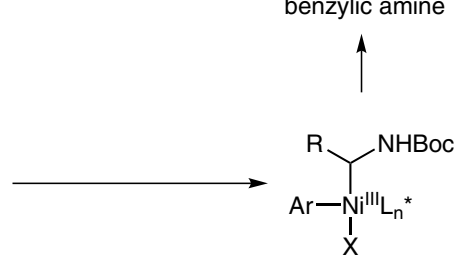<smiles>[2H]C([NH3+])c1ccccc1</smiles>
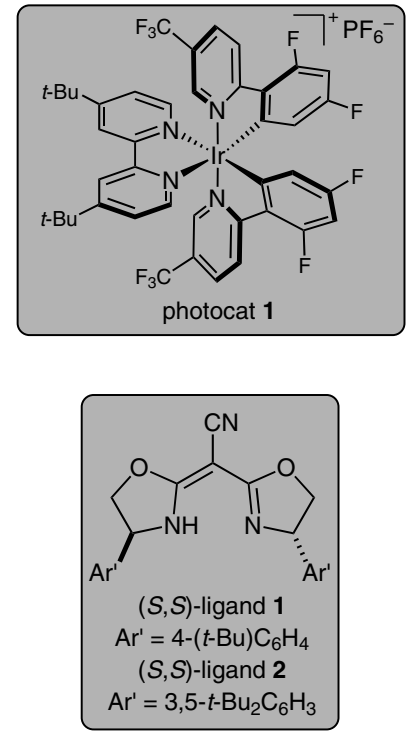

Category

Metal-Catalyzed Asymmetric Synthesis and Stereoselective Reactions

\section{Key words}

asymmetric catalysis

coupling reaction

photoredox

nickel

decarboxylation

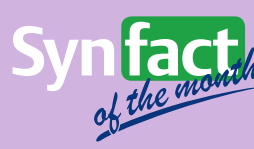

Selected examples:<smiles>CC(C)CC(N)c1ccc(C#N)cc1</smiles>

$71 \%$ yield, $92 \%$ ee<smiles>CC(C)CC(N)c1ccc(C#N)c(F)c1</smiles>

$76 \%$ yield, $91 \%$ ee<smiles>COC(=O)CC(N)c1ccc(C#N)cc1</smiles>

$80 \%$ yield, $82 \%$ ee

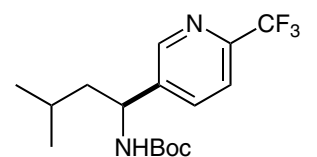

$73 \%$ yield, $92 \%$ ee<smiles>N#Cc1ccc(C(Cc2ccccc2)NC(=O)OCc2ccccc2)cc1</smiles>

$67 \%$ yield, $90 \%$ ee [using $(S, S)$-ligand 2 (2.2 mol\%) without $\left.n-\mathrm{Bu}_{4} \mathrm{NI}\right]$<smiles>CC(C)CC(NC(=O)OCc1ccccc1)c1cc(C(F)(F)F)cc(C(F)(F)F)c1</smiles>

$76 \%$ yield, $96 \%$ ee<smiles>N#Cc1ccc(C(Cc2cccs2)NC(=O)OCc2ccccc2)cc1</smiles>

$72 \%$ yield, $91 \%$ ee [using $(S, S)$-ligand 2 (2.2 $\mathrm{mol} \%$ ) without $\left.n-\mathrm{Bu}_{4} \mathrm{NI}\right]$<smiles>COC(=O)c1ccc(C(CC(C)C)NC(=O)OCc2ccccc2)cc1</smiles>

$69 \%$ yield, $90 \%$ ee
Significance: The authors present an asymmetric arylation of $\alpha$-amino acids by using a dual photoredox/chiral nickel catalysis approach. A broad array of naturally occurring $\alpha$-amino acids were arylated with various aryl halides to generate highvalue chiral benzylic amines in high yields and with high enantioselectivities.

sYNFACTS Contributors: Hisashi Yamamoto, Ramesh C. Samanta Synfacts 2016, 12(4), 0387 Published online: 15.03.2016 Dol: 10.1055/s-0035-1561772; Reg-No.: H01916SF
Comment: The authors have previously reported the coupling of aryl halides with an $\alpha$-carboxy $\mathrm{sp}^{3}$ carbon by dual photoredox reaction/nickel catalysis (Science 2014, 345, 437). Identification of chiral ligands compatible with a nickel catalyst under photoredox conditions permitted an asymmetric transformation. Chiral benzylamines that form the core structures of many pharmaceutically active molecules have been synthesized in optically pure form by this method. 